\title{
Effect of Treatment With Sodium-Glucose Cotransporter 2 Inhibitor on the Initiation of Continuous Positive Airway Pressure Therapy in Type 2 Diabetic Patients With Obstructive Sleep Apnea Syndrome
}

\author{
Masataka Kusunoki a, f, Fumiya Hisano ${ }^{\mathrm{b}}$, Naomi Wakazono ${ }^{\mathrm{a}}$, Kazuhiko Tsutsumi ${ }^{\mathrm{c}}$, \\ Yoshiharu Oshida ${ }^{\mathrm{d}}$, Tetsuro Miyata ${ }^{\mathrm{e}}$
}

\begin{abstract}
Background: Obese patients with type 2 diabetes mellitus often develop obstructive sleep apnea syndrome (OSAS). In this study, continuous positive airway pressure (CPAP) was initiated in Japanese patients with type 2 diabetes mellitus who developed OSAS during treatment with a sodium-glucose cotransporter 2 (SGLT2) inhibitor, and the effect of the SGLT2 inhibitor therapy on the patients was investigated.

Methods: The study was conducted in outpatients with type 2 diabetes mellitus with serum hemoglobin $\mathrm{A} 1 \mathrm{c}$ ( $\mathrm{HbA} 1 \mathrm{c})$ values of $\geq 6.5 \%$ who developed OSAS. The patients were divided into two groups according to whether they were receiving treatment with an SGLT2 inhibitor or with other oral hypoglycemic agents: the SGLT2 inhibitor group $(n=9)$ and non-SGLT2 inhibitor group $(n=7)$. The patients in the former group were under treatment with one of the following three SGLT2 inhibitors: luseogliflozin ( $2.5 \mathrm{mg}$ /day), dapagliflozin (5 $\mathrm{mg} /$ day) and empagliflozin $(10 \mathrm{mg} /$ day $)$. The patients took the drugs once daily, before or after breakfast. The patients were initiated on CPAP therapy for OSAS, and their weight, body mass index (BMI), serum $\mathrm{HbA1c}$ level, lipid profile, liver function parameters, serum uric acid, and apnea-hypopnea index (AHI) measured before the initiation of CPAP therapy (baseline) were compared with the values measured 3 months after the start of CPAP therapy.
\end{abstract}

Manuscript submitted October 25, 2021, accepted November 9, 2021 Published online November 20, 2021

aDepartment of Diabetes, Motor Function and Metabolism, Research Center of Health, Physical Fitness and Sports, Nagoya University, Nagoya, Japan

${ }^{b}$ Graduate School of Medicine, Department of Integrated Health Sciences, Nagoya University, Nagoya, Japan

'Okinaka Memorial Institute for Medical Research, Tokyo, Japan

${ }^{\mathrm{d}}$ Medical Checkup Center, Minami Seikyo Hospital, Nagoya, Japan

eOffice of Medical Education, School of Medicine, International University of Health and Welfare, Chiba, Japan

${ }^{\mathrm{f}}$ Corresponding Author: Masataka Kusunoki, Department of Diabetes, Motor Function and Metabolism, Research Center of Health, Physical Fitness and Sports, Nagoya University, Furou-cho, Chigusa-ku, Nagoya City, Aichi 4640814, Japan. Email: kusu0910noki@yahoo.co.jp

doi: https://doi.org/10.14740/jocmr4626
Results: The AHI decreased significantly after 3 months of CPAP therapy, as compared to that at the baseline, in both the SGLT2 inhibitor and non-SGLT2 inhibitor groups. There was no significant change in the serum HbA1c value after 3 months of CPAP therapy as compared to that at the baseline in either group. The body weight and BMI increased significantly after 3 months of CPAP therapy in the SGLT2 inhibitor group, but not in the non-SGLT2 inhibitor group.

Conclusion: The body weight and BMI increased significantly after 3 months of CPAP therapy initiated for OSAS in the type 2 diabetic patients who were receiving SGLT2 inhibitor therapy. Thus, when CPAP therapy is adopted for an obese diabetic patient with OSAS, it should be borne in mind that the body weight may increase if the patient is receiving SGLT2 inhibitor treatment.

Keywords: Type 2 diabetes mellitus; SGLT2 inhibitor; OSAS; CPAP; Body weight; HbA1c

\section{Introduction}

Type 2 diabetic patients with obesity often also develop obstructive sleep apnea syndrome (OSAS), suggesting that deterioration of the blood glucose and body weight is associated with an elevated risk of development of OSAS $[1,2]$. In addition, development of OSAS has been reported to exacerbate insulin resistance in patients with type 2 diabetes mellitus [3]. OSAS increases the cardiovascular risk and lowers the quality of life of the patients [4]. Improvement of the blood glucose and body weight is considered to be necessary to improve the condition in patients with OSAS. Sodium-glucose cotransporter 2 (SGLT2) inhibitors exert their hypoglycemic effect in patients with type 2 diabetes mellitus by selectively inhibiting SGLT2 to reduce the renal tubular reabsorption of glucose, thereby increasing urinary excretion of the excess blood glucose [5]. In addition to their hypoglycemic effect, SGLT2 inhibitors have also been reported to reduce the body weight, lower the blood pressure, improve lipid metabolism and uric acid metabolism, and ameliorate liver dysfunction [6, 7]. Through these pleiotropic effects, SGLT2 inhibitors have been reported to signifi- 
cantly reduce the cardiovascular risk in patients with type 2 diabetes mellitus and elevated cardiovascular risk [8]. In this study, the effects of SGLT2 inhibitor therapy versus treatment with other hypoglycemic agents were evaluated in Japanese patients with type 2 diabetes mellitus who were initiated on continuous positive airway pressure (CPAP) therapy for OSAS developing during treatment with SGLT2 inhibitors.

\section{Materials and Methods}

This study was conducted in compliance with the ethical standards of the responsible institution for studies in human subjects as well as with the Declaration of Helsinki. The protocol of this study was reviewed and approved by the institutional review board of the Medical Corporation Odakai Ethics Committee (IRB approval number 2014-03).

\section{Subjects}

Japanese type 2 diabetic patients with serum hemoglobin A1c (HbA1c) values of $\geq 6.5 \%$ who developed OSAS during treatment with antidiabetic drugs were enrolled in this study. Consent for participation in the study was obtained from the subjects after they had received an explanation about the purpose of the study from their physicians. This prospective clinical study is officially registered as an open-label study (ID: UMIN000045226).

\section{Study methods}

Patients with type 2 diabetes mellitus who developed OSAS during treatment with oral antidiabetic drugs and were initiated on CPAP therapy were enrolled in this study; only those patients in whom the blood glucose levels had been stabilized by treatment with the same hypoglycemic agents and no new drugs had been added for $\geq 6$ months $(n=16,14$ males and two females) were eligible. The subjects were divided into two groups according to whether they were receiving treatment with an SGLT2 inhibitor or with other hypoglycemic agents: the SGLT2 inhibitor group, comprising type 2 diabetic subjects who were receiving treatment with an SGLT2 inhibitor ( $\mathrm{n}=9$, seven males and two females), and the non-SGLT2 inhibitor group, comprising type 2 diabetic subjects who were receiving treatment with hypoglycemic agents other than an SGLT2 inhibitor ( $\mathrm{n}=7$, seven males and none female).

Subjects of the SGLT2 inhibitor group in this study were receiving any one of the following three SGLT2 inhibitors: luseogliflozin (Taisho Pharmaceutical Holdings Co., Ltd, Tokyo, Japan), dapagliflozin (Astra Zeneca, Osaka, Japan), and empagliflozin (Boehringer Ingelheim Japan, Inc., Tokyo, Japan). The subjects took the drugs once daily before or after breakfast at the following doses: luseogliflozin, $2.5 \mathrm{mg} /$ day; dapagliflozin, $5 \mathrm{mg}$ /day; empagliflozin, $10 \mathrm{mg} /$ day. Each patient received one of the three SGLT2 inhibitors.

The hypoglycemic agents used for treatment in the non-
SGLT2 inhibitor group included dipeptidyl peptidase 4 (DPP4) inhibitors, biguanides, sulfonylureas, $\alpha$-glucosidase inhibitors, or thiazolidinediones. In addition, some patients from both groups were receiving two or more drugs with different mechanisms of action. Some of the study subjects were also receiving lipid-lowering drugs and/or antihypertensive drugs.

The patients were initiated on CPAP therapy (ResMed Inc., San Diego, USA) at home for the treatment of OSAS for 3 months. The CPAP was performed according to the compliance of CPAP issued by Japan Society for Study of Obesity.

The AHI was measured using the sleep assessment device PulSleep LS-140 (FUKUDA DENSHI, Inc., Tokyo, Japan).

Blood samples for various laboratory measurements were collected before the initiation of CPAP therapy (on the day before the start of treatment) and after 3 months of CPAP therapy. Measurements of the serum HbAlc, aspartate aminotransferase (AST), alanine aminotransferase (ALT), $\gamma$-glutamyl transpeptidase $(\gamma-\mathrm{GTP})$, uric acid, and blood lipid levels were entrusted to Handa Medical Association Health Center (Aichi, Japan); serum AST, ALT, $\gamma$-GTP, and uric acid and blood lipid levels were measured using an autoanalyzer (JCA-BM8000 series, JAOL, Tokyo, Japan), and the serum HbA1c values were measured using an automated HPLC assay (HLC-723GX, Tosoh Corporation, Tokyo, Japan). Paired $t$-test was used to test the significance of differences; the level of significance was set at $\mathrm{P}<0.05$.

\section{Results}

\section{Patient characteristics}

Table 1 shows the patient characteristics.

Effects of SGLT2 inhibitors on the body weight, BMI, serum HbA1c value, blood lipid profile, serum uric acid level, liver function parameters, and AHI

There were no statistical differences in all baseline values between two groups.

Significant increases of the body weight and BMI were observed at the end of 3 months of CPAP therapy, as compared to the values recorded at the baseline, in the SGLT2 inhibitor group. No significant change of the body weight or BMI was observed, as compared to the baseline, after 3 months of CPAP therapy in the non-SGLT2 inhibitor group.

No significant changes of the serum $\mathrm{HbAlc}$ value were observed after 3 months of CPAP therapy in either group.

No significant changes in liver function parameters, including the serum levels of AST, ALT or $\gamma$-GTP, were observed after 3 months of CPAP therapy in either group.

Furthermore, no significant changes in the blood lipid profile, namely, the serum levels of total cholesterol, high-density lipoprotein cholesterol (HDL-C), low-density lipoprotein cholesterol (LDL-C) or triglycerides, were found after 3 months of CPAP therapy in either group.

There was also no change in the serum uric acid level after 
Table 1. Patient Characteristics at Baseline

\begin{tabular}{lll}
\hline Group & $\begin{array}{l}\text { SGLT2 } \\
\text { inhibitor }\end{array}$ & $\begin{array}{l}\text { Non-SGLT2 } \\
\text { inhibitor }\end{array}$ \\
\hline $\mathrm{n}$ & 9 & 7 \\
Male/female & $7 / 2$ & $7 / 0$ \\
Age (years) & $59 \pm 12$ & $55 \pm 9$ \\
Body weight (kg) & $80.2 \pm 17.1$ & $90.3 \pm 13.4$ \\
BMI (kg/m $\left.{ }^{2}\right)$ & $28.0 \pm 5.4$ & $29.5 \pm 3.6$ \\
HbA1c (\%) & $6.9 \pm 0.5$ & $7.1 \pm 0.7$ \\
Concomitant medication & & \\
$\quad$ Antidiabetic drug (n) & & \\
$\quad$ DPP4 inhibitor & 8 & 4 \\
$\quad$ Biguanide & 6 & 2 \\
$\quad$ Sulfonylurea & 1 & 1 \\
$\quad$ alpha-glucosidase inhibitor & 1 & 1 \\
$\quad$ Thiazolidinedione & 4 & 2 \\
\hline Lipid-modifying agents (n) & 9 & 2 \\
Antihypertensive drug (n) & 1 & 4 \\
\hline
\end{tabular}

SGLT2: sodium-glucose cotransporter 2; BMI: body mass index HbA1c: hemoglobin A1c; DPP4: dipeptidyl peptidase 4; SD: standard deviation. Data are expressed as means \pm SD.

3 months of CPAP therapy in either group.

The AHI decreased significantly after 3 months of CPAP therapy in both groups.
Regarding days of CPAP performed per month, there was no difference between two groups (Table 2).

\section{Discussion}

OSAS has been reported to increase the risk of development of diabetes mellitus, hypertension, and heart disease $[4,9]$. Furthermore, treatment of OSAS by CPAP therapy has been reported to reduce the risk of development of these diseases $[9,10]$. However, few studies have investigated the effects of hypoglycemic agents on the glycemic control and body weight during CPAP therapy for OSAS. The purpose of this study was to investigate the effects of SGLT2 inhibitor therapy in patients with type 2 diabetes mellitus initiated on CPAP therapy for OSAS, by comparing the changes in the values of relevant parameters measured before and 3 months after the initiation of CPAP therapy between patients receiving SGLT2 inhibitor therapy (SGLT2 inhibitor group) and those receiving other classes of oral hypoglycemic agents (non-SGLT2 inhibitor group).

Significant decrease of the AHI and improvement of OSAS was observed following 3 months of CPAP therapy in both the SGLT2 inhibitor group and non-SGLT2 inhibitor group.

The body weight and BMI increased significantly after 3 months of CPAP therapy, as compared to the baseline, in the SGLT2 inhibitor group; on the other hand, CPAP therapy for 3 months had no effect on the body weight or BMI in the nonSGLT2 inhibitor group.

SGLT2 inhibitors have been reported to reduce the body weight and BMI in patients with type 2 diabetes mellitus $[6$,

Table 2. Effects of SGLT2 Inhibitors on Various Parameters in Type 2 Diabetic Patients With OSAS

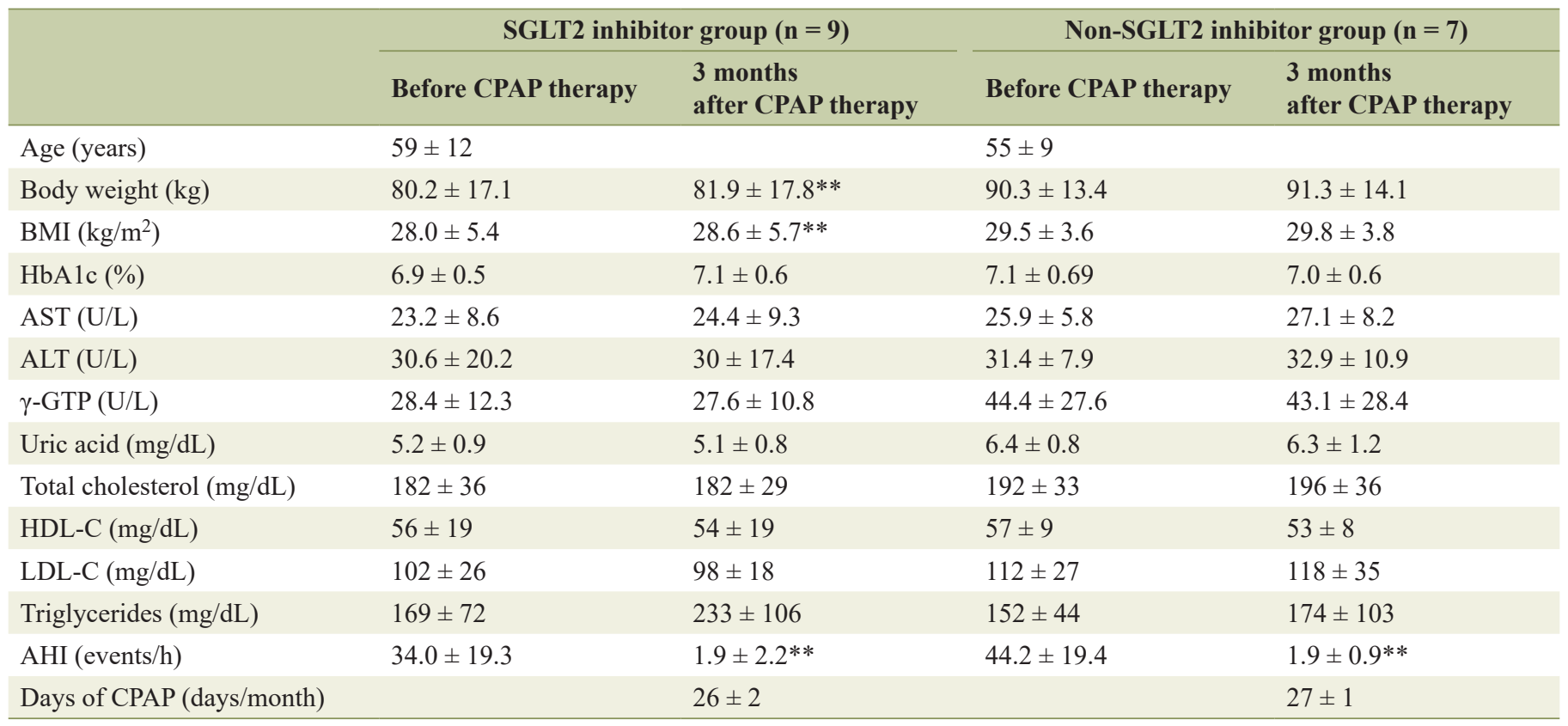

Data are expressed as means $\pm \mathrm{SD}$. ${ }^{*} \mathrm{P}<0.05$, ${ }^{*} \mathrm{P}<0.01 \mathrm{vs}$. before CPAP therapy. Days of CPAP: days of performed per month. SGLT2: sodiumglucose cotransporter 2; OSAS: obstructive sleep apnea syndrome; CPAP: continuous positive airway pressure; BMI: body mass index; HbA1c: hemoglobin A1c; AST: aspartate aminotransferase; ALT: alanine aminotransferase; y-GTP: y-glutamyl transpeptidase; LDL-C: low-density lipoprotein cholesterol; HDL-C: high-density lipoprotein cholesterol; AHI: apnea-hypopnea index 
7]. Sawada et al reported that administration of SGLT2 inhibitors reduced the body weight and BMI in type 2 diabetic patients with OSAS who had not been initiated on any treatment for OSAS [11]. The weight loss in patients receiving SGLT2 inhibitors is thought to be the result of calorie loss (reduced calorie supply) associated with the excess urinary glucose excretion [12].

In our study, SGLT2 inhibitors had been administered continuously for 6 months prior to the initiation of CPAP therapy for the treatment of OSAS, suggesting that the body weight had decreased during the period from the start of administration of SGLT2 inhibitor treatment 6 months prior to the study until the initiation of CPAP therapy (on the day before the start of treatment). Therefore, the weight gain after the initiation of CPAP therapy for OSAS may be considered as being due to the improvement of the OSAS following the initiation of CPAP therapy. The increase in body weight is possible as a rebound after weight loss in patients treated with SGLT2 inhibitors. However, Bolinder et al reported that body weight level decreased in response to dapagliflozin treatment and this effect continued until over 2 years [13].

In contrast to the belief that SGLT2 inhibitor treatment results in weight loss, an animal study conducted in rats reported that administration of SGLT2 inhibitors was associated with increased food intake by the animals [14]. In addition, Chiba et al found that administration of the SGLT2 inhibitor dapagliflozin reduced energy consumption in mice, and reported that this was caused by reduced expression of uncoupling protein 1 (UCP1) in the brown adipocytes due to decreased sympathetic activity [15]. However, our search of the literature yielded no clinical reports on the effects of SGLT2 inhibitors on the sympathetic nervous system or the appetite.

In this study, patients receiving SGLT2 inhibitor therapy who developed OSAS and were initiated on CPAP therapy showed increase of the body weight after 3 months of CPAP therapy; this was speculated to be attributable to the improved appetite associated with improvement of the OSAS, along with the appetite-enhancing effect and sympathetic nervous activity-lowering effect of SGLT2 inhibitors. However, future studies with measurement of the dietary intakes in patients receiving SGLT2 inhibitor therapy are required to elucidate the precise cause of the weight gain. It is also necessary to clarify the clinical effects of SGLT2 inhibitor therapy on the sympathetic nervous system, parasympathetic nervous system and the feeding centers of the brain.

Brooks et al reported that CPAP therapy improved the insulin sensitivity in diabetic patients with OSAS [16]. In the present study, no change in the serum level of $\mathrm{HbA1c}$, which is an indicator of glycemic control, was found after 3 months of CPAP therapy in either the SGLT2 inhibitor group or the nonSGLT2 inhibitor group. SGLT2 inhibitors have been reported to improve the serum levels of AST, ALT and $\gamma$-GTP, which are liver function parameters, and also improve the blood lipid profile and decrease the serum uric acid level $[6,7]$. However, in the present study, no changes in any of these aforementioned parameters were found after 3 months of CPAP therapy in either study group. This is considered to be because all the patients had received the hypoglycemic agents continuously for a period of at least 6 months before the initiation of CPAP therapy and the effects of the hypoglycemic agents on the parameters mentioned abovemay have already reached a peak before the initiation of the CPAP therapy (on the day before the start of treatment).

\section{Conclusion}

In this study, type 2 diabetic patients receiving SGLT2 inhibitor therapy who developed OSAS were initiated on CPAP therapy. While no significant effect on the serum $\mathrm{HbAlc}$ value was observed, the body weight and BMI of the patients increased after 3 months of CPAP therapy. Thus, when a type 2 diabetic patient on SGLT2 inhibitor therapy develops OSAS and needs to be initiated on CPAP therapy, it must be borne in mind that his/her weight could increase after the start of the CPAP therapy and that measures for weight control might need to be implemented.

\section{Acknowledgments}

There is no specific acknowledgment that warrants mention.

\section{Financial Disclosure}

The research did not receive any specific grant from funding agencies in the public, commercial, or not for profit sectors.

\section{Conflict of Interest}

The authors declare that they have no financial or non-financial conflict of interest to report.

\section{Informed Consent}

Informed consent was obtained.

\section{Author Contributions}

MK has designed and performed the study. MK, KT and TM have drafted the manuscript and did critical editing. NW, FH and YO have assisted and supported in data collection and subsequent analysis with statistics.

\section{Data Availability}

Any inquiries regarding the availability of the supporting data from this study should be directed to the corresponding author.

\section{Abbreviations}

SGLT2: sodium-glucose cotransporter 2; OSAS: obstructive 
sleep apnea syndrome; CPAP: continuous positive airway pressure; AHI: apnea-hypopnea index; BMI: body mass index; HbA1c: hemoglobin A1c; AST: aspartate aminotransferase; ALT: alanine aminotransferase; $\gamma$-GTP: $\gamma$-glutamyl transpeptidase; UCP1: uncoupling protein 1; LDL-C: low-density lipoprotein cholesterol; HDL-C: high-density lipoprotein cholesterol

\section{References}

1. Sasanabe R, Banno K, Otake K, Hasegawa R, Usui K, Morita M, Shiomi T. Metabolic syndrome in Japanese patients with obstructive sleep apnea syndrome. Hypertens Res. 2006;29(5):315-322.

2. Bonsignore MR, Borel AL, Machan E, Grunstein R. Sleep apnea and metabolic dysfunction. Euro Resp Rev 2013; 22(129):353-364.

3. Aurora RN, Punjabi NM. Obstructive sleep apnoea and type 2 diabetes mellitus: a bidirectional association. Lancet Respir Med. 2013;1(4):329-338.

4. Shamsuzzaman AS, Gersh BJ, Somers VK. Obstructive sleep apnea: implications for cardiac and vascular disease. JAMA. 2003;290(14):1906-1914.

5. DeFronzo RA, Davidson JA, Del Prato S. The role of the kidneys in glucose homeostasis: a new path towards normalizing glycaemia. Diabetes Obes Metab. 2012;14(1):514.

6. Seino Y, Sasaki T, Fukatsu A, Ubukata M, Sakai S, Samukawa Y. Efficacy and safety of luseogliflozin as monotherapy in Japanese patients with type 2 diabetes mellitus: a randomized, double-blind, placebo-controlled, phase 3 study. Curr Med Res Opin. 2014;30(7):1245-1255.

7. Kusunoki M, Natsume Y, Sato D, Tsutsui H, Miyata T, Tsutsumi K, Suga T, et al. Luseogliflozin, a sodium glucose co-transporter 2 inhibitor, alleviates hepatic impairment in japanese patients with type 2 diabetes. Drug Res (Stuttg). 2016;66(11):603-606.

8. Zinman B, Wanner C, Lachin JM, Fitchett D, Bluhmki E, Hantel S, Mattheus M, et al. Empagliflozin, cardiovascular outcomes, and mortality in type 2 diabetes. N Engl J Med. 2015;373(22):2117-2128.

9. Marin JM, Carrizo SJ, Vicente E, Agusti AG. Long-term cardiovascular outcomes in men with obstructive sleep apnoea-hypopnoea with or without treatment with continuous positive airway pressure: an observational study. Lancet. 2005;365(9464):1046-1053.

10. Steiropoulos P, Tsara V, Nena E, Fitili C, Kataropoulou M, Froudarakis M, Christaki P, et al. Effect of continuous positive airway pressure treatment on serum cardiovascular risk factors in patients with obstructive sleep apneahypopnea syndrome. Chest. 2007;132(3):843-851.

11. Sawada K, Karashima S, Kometani M, Oka R, Takeda Y, Sawamura T, Fujimoto A, et al. Effect of sodium glucose cotransporter 2 inhibitors on obstructive sleep apnea in patients with type 2 diabetes. Endocr J. 2018;65(4):461467.

12. Nauck MA, Del Prato S, Meier JJ, Duran-Garcia S, Rohwedder K, Elze M, Parikh SJ. Dapagliflozin versus glipizide as add-on therapy in patients with type 2 diabetes who have inadequate glycemic control with metformin: a randomized, 52-week, double-blind, active-controlled noninferiority trial. Diabetes Care. 2011;34(9):20152022.

13. Bolinder J, Ljunggren O, Johansson L, Wilding J, Langkilde AM, Sjostrom CD, Sugg J, et al. Dapagliflozin maintains glycaemic control while reducing weight and body fat mass over 2 years in patients with type 2 diabetes mellitus inadequately controlled on metformin. Diabetes Obes Metab. 2014;16(2):159-169.

14. Devenny JJ, Godonis HE, Harvey SJ, Rooney S, Cullen MJ, Pelleymounter MA. Weight loss induced by chronic dapagliflozin treatment is attenuated by compensatory hyperphagia in diet-induced obese (DIO) rats. Obesity (Silver Spring). 2012;20(8):1645-1652.

15. Chiba Y, Yamada T, Tsukita S, Takahashi K, Munakata Y, Shirai Y, Kodama S, et al. Dapagliflozin, a sodiumglucose co-transporter 2 inhibitor, acutely reduces energy expenditure in BAT via neural signals in mice. PLoS One. 2016;11(3):e0150756.

16. Brooks B, Cistulli PA, Borkman M, Ross G, McGhee $\mathrm{S}$, Grunstein RR, Sullivan CE, et al. Obstructive sleep apnea in obese noninsulin-dependent diabetic patients: effect of continuous positive airway pressure treatment on insulin responsiveness. J Clin Endocrinol Metab. 1994;79(6):1681-1685. 